Chikungunya Fever: long lasting burden of an acute illness. BMC Infectious Diseases 2010 10:31.

Disclosure of Interest: None declared

DOI: 10.1136/annrheumdis-2017-eular.5554

\section{SAT0567 IMPACT OF CHIKUNGUNYA FEVER ON FUNCTIONAL STATUS AND QUALITY OF LIFE - A PROSPECTIVE COHORT STUDY OF BRAZILIAN PATIENTS}

L.F. da Rocha Jr ${ }^{1,2}$, M.R.D.A. Freitas ${ }^{1}$, A.G.L. de Mattos ${ }^{1}$, H.D. de Lima ${ }^{1}$ R.M. Correia ${ }^{1}$, F.G.T. Vieira ${ }^{1}$, A.L.B.P. Duarte ${ }^{2}$, C.D.L. Marques ${ }^{2}$, W.L.M. de Oliveira $^{1}$, P.R.S. de Melo ${ }^{1}$, A. Ranzolin ${ }^{1,2} .{ }^{1}$ Instituto de Medicina Integral Professor Fernando Figueira; ${ }^{2}$ Hospital Das Clínicas of Pernambuco, Recife, Brazil

Background: An epidemic of Chikungunya Fever (CF) spread throughout South America in 2014. The acute manifestation of CF typically consists of febrile arthritis. The burden of the chronic articular manifestations remains a public health issue affecting activities of daily life. There is a very important impact on quality of life in patients affected by CF, even at chronic phase. The long-term functional status may also be affected by CF.

Objectives: To evaluate longitudinally the disability, Health Related Quality of Life (HRQOL) and functional status of patients with CF and analyze the clinical and epidemiological factors associated with different outcomes.

Methods: Patients with clinical and demographic diagnosis of CF and persistent articular symptoms were evaluated in a cohort study between May 2016 and December 2016. HRQOL was rated by Short-Form 12 (SF-12) and the functional status was checked through Health Assessment Questionnaire (HAQ) and the Global Functional Status (GFS). Data were divided per weeks after disease onset and were analysed (Spearmans's correlation coefficient and Mann-Whitney test). Results: Sixty-five patients (58 females), mean age of $51.3( \pm 13.3)$ were assessed. As expected, a significant correlation between pain related scores and Physical Health Composite Scale Score (PCS), HAQ and GFS was found $(p<0.05))$. Edema and morning stiffness correlated with PCS, HAQ and GFS status from 4 to 20 weeks after disease onset $(p<0.05)$. There was improvement in scores of all instruments used from 4-8 weeks of disease to 12-16 weeks of disease (table 1). The worst indices of PCS, Mental Health Composite Scale Score (MCS) and GFS were scored in the first month, mean scores of $30.07 \pm 5.77$, $38.13 \pm 8.54$ and $3.15 \pm 1.07$ respectively. Higher $\mathrm{HAQ}$ values were demonstrated between 4 and 8 weeks after disease onset (mean score 1.87 \pm 0.82 ).

HRQOL and Functional Status in patients with CF

\begin{tabular}{lccc}
\hline & $\begin{array}{c}4-8 \text { weeks of disease } \\
\text { (mean score) }\end{array}$ & $\begin{array}{c}\text { 12-16 weeks of disease } \\
\text { (mean score) }\end{array}$ & P value \\
\hline PCS & $30.12 \pm 8.21$ & $35.86 \pm 11.11$ & 0.0487 \\
MCS & $40.95 \pm 12.23$ & $47.02 \pm 12.09$ & 0.0326 \\
HAQ & $1.87 \pm 0.82$ & $1.36 \pm 0.86$ & 0.0228 \\
Global Functional Status & $3.03 \pm 0.98$ & $2.53 \pm 0.95$ & 0.0438 \\
\hline
\end{tabular}

Conclusions: We demonstrated the impact of CF on HRQOL and Functional Status of patients. The SF-12 Health Survey, HAQ and GFS are influenced mostly by patients pain and worsening of this status are more prominent in the first 8 weeks of disease. Further clinical studies of the impact of CF on quality of life and functional studies are needed

\section{References:}

[1] Soumahoro MK, Gérardin P, Boëlle PY et al. Impact of Chikungunya virus infection on health status and quality of life: a retrospective cohort study. PLoS One. 2009 Nov 11;4(11):e7800.

[2] Rahim AA, Thekkekara RJ, Bina $T$ et al. Disability with Persistent Pain Following an Epidemic of Chikungunya in Rural South India. J Rheumatol. 2016 Feb;43(2):440-4.

[3] Couturier E, Guillemin F, Mura M et al. Impaired quality of life after chikungunya virus infection: a 2-year follow-up study. Rheumatology (Oxford). 2012 Jul;51(7):1315-22.

Disclosure of Interest: None declared

DOI: 10.1136/annrheumdis-2017-eular.5604

\section{SAT0568 RHEUMATOLOGICAL MANIFESTATIONS IN A SERIES OF PATIENTS WITH CHIKUNGUNYA FEVER}

A.M. Sapag Duran ${ }^{1}$, A.M. Beron ${ }^{2}$, S. Sapag Duran ${ }^{2}$, C. Coral Cristado ${ }^{1}$, G. Medina ${ }^{2}$, G. Nasswetter ${ }^{2}$, D. Dubinsky ${ }^{2} .{ }^{1}$ Rheumatology, Hospital Universitario Japones, Santa Cruz, Bolivia, Plurinational State Of;

${ }^{2}$ Rheumatology, Clinical Hospital "Jose de San Martin", University of Buenos Aires., CABA, Argentina

Background: Chikungunya fever is characterised by a high probability of persistent rheumatological manifestations, producing a negative impact in the work, social and economic fields.

Objectives: To determine the frequency and type of rheumatologic involvement in the subacute and chronic phase of Chikungunya fever.

Methods: Descriptive, cross-sectional study. We included patients $>16$ years old with Chikungunya infection (real time PCR, IgM or IgG for Chikungunya) who consulted consecutively for rheumatic symptoms/signs from March 2015 to March 2016. According to the time of evolution, the disease was divided in 2 Phases: acute ( $\leq 10$ days of duration) and subacute/chronic ( $\geq 11$ days). According to clinical presentation, patients were classified in two groups: 1) non-autoimmune rheumatologic compromise (NARC) and 2) autoimmune rheumatologic compromise (ARC). Current ACR/EULAR criteria for classification of autoimmune diseases were used.

Results: Two hundred and two patients were evaluated, 80 were excluded due to negative serology for Chikungunya. 122 were included: 107 (88\%) female, mean age $52.52 \pm 13.19$ years, and time of evolution of $116.66 \pm 91.61$ days.

Acute phase. 122 patients: fever $85(69.67 \%)$, rash and pruritus 54 (44.26\%), tenosynovitis $23(18.8 \%)$, polyarthralgias $100(82 \%)$ and arthritis $56(45.90 \%)$.

Chronic phase. 122 patients: 71 (58\%) patients had a chronic persistent rheumatologic symptoms and $51(42 \%)$ presented remission of symptoms but all of them presented subsequent recurrence in an $91 \pm 40$ days. NARC in 33 patients $(27 \%)$ and ARC in $89(73 \%)$, with no significant differences in age and time of evolution was observed.

NARC: $14(42.4 \%)$ exacerbation of previous osteoarthritis pain, $9(27.3 \%)$ developed fibromyalgia and $10(30.3 \%)$ had localized soft tissue pain.

ARC: $13(14.6 \%)$ with a history of RA, SLE, psoriasis or DM reactivated the underlying disease and $76(85.4 \%)$ developed ARC: Undifferentiated polyarthritis with negative antibodies $61(80 \%)$, RA with positive antibodies $5(6.5 \%)$, scleroderma $2(2.6 \%)$, cutaneous vasculitis $2(2.6 \%)$, polymyalgia rheumatica 1 (1.3\%), Sjogren's Syndrome 2 (2.6\%), Dermatomyositis 1, Erythema nodosum 1 $(1.3 \%)$ and vitiligo $1(1.3 \%)$.

Antibodies were requested according to clinical suspicion: $F A N \geq 320$ in 5 patients, $\mathrm{RF}$ in 6, ACPA in 4 and anti RO in 1. Thyroid dysfunction was observed in 7 patients who had a previous normal thyroid profile.

\begin{tabular}{lccc}
\hline & Acute Fase $\mathrm{n} 122(\%)$ & Chronic Fase $\mathrm{n} 122(\%)$ & $\mathrm{p}$ \\
\hline Fever & $85(69,7)$ & 0 & 0,01 \\
Rash and pruritus & $54(44,2)$ & 0 & 0,01 \\
Tenosynovitis & $23(18,8)$ & $41(33,61)$ & NS \\
Polyarthralgias & $100(82)$ & $83(68,03)$ & NS \\
Arthritis & $56(45.9)$ & $81(66,39)$ & 0,0005 \\
\hline
\end{tabular}

Conclusions: The frequency of rheumatological manifestations post Chikungunya fever in our sample was high, and can trigger ARC. Patients presenting new immunological manifestations in an endemic area for Chikungunya fever should have a serologic test performed. This series of patients must be evaluated with long-term studies to define their evolution, under the possibility of developing definite autoimmune disease or remission.

References:

[1] Chikungunya Pathology and Cytokines. Chow et al. JID 2011.

Disclosure of Interest: None declared

DOI: 10.1136/annrheumdis-2017-eular.2755

\section{SAT0569 OUTCOME OF PATIENTS WITH SYSTEMIC RHEUMATIC DISEASES ADMITTED IN INTENSIVE CARE UNIT: A PROGNOSTIC STUDY OF 98 PATIENTS}

A. Malézieux-Picard $^{1}$, H. Hivernat ${ }^{2}$, N. Martis ${ }^{1}$, J.-G. Fuzibet ${ }^{1}$, G. Bernardin ${ }^{2}$, V. Queyrel ${ }^{1} .{ }^{1}$ Internal medecine departement; ${ }^{2}$ Intensive care unit, Centre Hospitalier Universitaire de Nice, Nice, France

Background: Systemic rheumatic diseases (SRD) are a rare and heterogeneous group of diseases, associated with a high mortality rate due to the natural evolution of the disease and/or consequences of their specific treatments (infections, toxicity)

Objectives: To describe the clinical features, outcomes and prognostic factors for patients with SRD admitted to the intensive care unit (ICU).

Methods: Single-center retrospective observational cohort study of 98 patients with SRD over an 11-year period in an ICU of a French teaching hospital.

Results: Ninety-eight patients (57\% women; median age, 57 years [19-81years]) accounted for 108 admissions. Connective tissue disease (primarily systemic lupus erythematosus) and systemic vasculitides (mainly ANCA-associated vasculitides) represented respectively $55 \%$ and $30 \%$ of SRD. For nineteen patients, diagnosis of SRD was made at admission. Reasons for admission were: SRD exacerbations $(43 \%)$, isolated infections (34\%), SRD exacerbations associated with infections $(12 \%)$ or other $(11 \%)$. Respiratory failure was the most common organ dysfunction. Mechanical ventilation was necessary for 43 patients (44\%), vasoactive drugs for 47 (48\%) and extra-renal replacement therapy for 38 (39\%). The ICU mortality rate was $30 \%$ and $37 \%$ one year after admission. Infection was the main cause of death (69\%). The factors significantly associated with mortality in the ICU were (multivariate analysis): diabetes, cardiovascular diseases and immunosuppressive treatments on admission. At 1 year of follow-up, additional risk factors were: number of organ dysfunction at ICU admission and mechanical ventilation. It is to be noted that at 1 year of follow-up, diabetes was not anymore a prognostic factor. Conclusions: Patients with SRD admitted to the ICU have a severe prognosis. Causes of mortality are mainly infections. Our study points out the importance of vaccination and developing new therapeutic strategies. Diagnosis of SRD in the ICU is not rare and should be systematically considered on admission. Prognostic factors of mortality in the ICU were patient comorbidities and immunosuppressive 
therapy at admission. In addition, mechanical ventilation and multiple organ failure were risk factors for mortality at 1 year.

Disclosure of Interest: None declared

DOI: 10.1136/annrheumdis-2017-eular.3692

\section{SAT0570 AN IGNORED DISEASE IN ADULTS: ACUTE RHEUMATIC FEVER}

B. Bitik $^{1}$, O. Kucuksahin ${ }^{2}$, N. Karahan Yesil ${ }^{1}$, H. Yesil ${ }^{2}$, S. Erten ${ }^{2}$ ${ }^{1}$ Rheumatology, Ankara Research and Education Hospital; ${ }^{2}$ Rheumatology, Yildirim Beyazit University, Ankara, Turkey

Background: Acute rheumatic fever (ARF) is a delayed, inflammatory sequela of pharyngitis secondary to Group A Streptococcus infection. ARF remains one of the most important causes of cardiovascular morbidity and mortality in developing countries. Although it is mainly known as a childhood disease, it is also encountered in adult clinics in developing countries.

Objectives: To investigate the clinical and laboratory characteristics of patients who were diagnosed with ARF in two rheumatology outpatient clinics from June 2015 to January 2017.

Methods: The data of 20 patients (12 female; median age 29.5 (21-40) years) were evaluated. The diagnosis of ARF was based on the 2015 Jones criteria. The data collected included patient age, gender, arthralgia, arthritis, erythema marginatum (EM), subcutaneous nodules (SN), ECG/ Doppler transthoracic echocardiography findings, and other rare findings. The erythrocyte sedimentation rate (ESR), antistreptolysin $\mathrm{O}$ (ASO) and CRP levels of the patients and the drugs initiated were also recorded. Anti-streptolysin O (ASO) test or throat culture were used for the evidence of preceding Streptococcus infection. Patients with post-streptococcal reactive arthritis were differentiated and excluded by clinically. Patients with positive rheumatoid factor or ACPA were also excluded. Joint fluid examination was done to exclude septic arthritis in patients with monoarthritis.

Results: All patients were referred to rheumatology for arthralgia or arthritis. Patients were taking some sort of nonsteroidal antiinflammatory (NSAI) drugs before the referral. The median follow-up time was 9 months $(0-18)$. Sixteen out of 20 patients had mono-, oligo- or poliarthritis (25\%,25\% and $30 \%$, respectively). Knees and ankles were the most common involved joints. The median duration of arthritis was 1 week (1-50 weeks). Six out 20 patients had subclinical carditis (30\%). Nine out of 20 patients had a history of ARF attack previously. Three patients had chronic rheumatic mitral valve thickening without any severe insufficiency. EM and SN were observed in $15 \%$ and $60 \%$ of patients, respectively. Chorea was diagnosed in one patient. NSAI drugs were given to all patients with maximum dosages. High dose salicylate therapy were not given to patients due to intolerance or side effects. Nine patients were given prednisolone therapy (5-20 $\mathrm{mg} / \mathrm{d}$ ). The median duration of prednisolone therapy was 2 weeks (0-6 weeks). Sulfasalazine was given to two patients for the prolonged arthritis. All patients received secondary prophylaxis with penicillin.

Conclusions: ARF should be considered in the differential diagnosis of arthritis in young adults in developing countries. Arthritis of ARF in adults seems to be resistant to classical NSAI drugs. Our data show that steroid therapy can be given safely instead of salicylates in carditis or arthritis.

\section{References:}

[1] GewitzMH,BaltimoreRS,TaniLY,etal. AmericanHeart Association Committee on Rheumatic Fever, Endocarditis and Kawasaki Disease of the Council on Cardiovascu- lar Disease in the Young. Revision of the Jones criteria for the diagnosis of the rheumatic fever in the era of Doppler echocardiography: a scientific statement of the American Heart Association. Circulation 2015; 131 : 1806-18.

[2] He VY, Condon JR, Ralph AP, et al. Long-Term Outcomes From Acute Rheumatic Fever and Rheumatic Heart Disease: A Data-Linkage and Survival Analysis Approach. Circulation. 2016 Jul 19;134(3):222-32.

Disclosure of Interest: None declared

DOI: 10.1136/annrheumdis-2017-eular.4944

\section{SAT0571 PERFORMING SEPTIC ARTHRITIS DIAGNOSIS IN SCENARIOS WHERE SYNOVIAL FLUID IS NOT AVAILABLE: MULTIVARIATE LOGISTIC REGRESSION ANALYSIS}

C.A. Guillen-Astete ${ }^{1}$, G.A. Gabilondo-Alvarez ${ }^{2} .{ }^{1}$ Rheumatology Department; ${ }^{2}$ Microbiology, Ramon y Cajal University Hospital, Madrid, Spain

Background: In order to establish the diagnosis of septic arthritis (SA) it is necessary to demonstrate the presence of bacteria into the synovial fluid. However, clinics should act according to its suspicious when the differential diagnosis included this possibility even when the microbiologic study of the synovial fluid in unavailable by any cause (small joints, lack of training or logistic deficiencies).

Objectives: The purpose of our study is to determine the relative weight of other clinical or analytical variables that should be useful in these scenarios.

Methods: A retrospective multivariate logistic regression analysis about the registries of monoarthritis assessed in our unit between 2013 and 2016. There were included only registries with cases of synovial joints with all parameters of interest available. The binary response variable was the microbiological demonstration of septic arthritis (culture/Gram stain of synovial fluid or tissue).
Explanatory variables were age (stratified in $<30,30-39,40-49,50-59,60-69$ and $>70$ years old), gender, temperature above of $37.9^{\circ} \mathrm{C}$, recount of neutrophils in peripheral blood sample, measure of procalcitonine (PCT), and measure of $C$ reactive protein (CRP). Synovial fluid study, although was available in almost all cases was deliberately omitted for purpose of this study. The logistic regression analysis used the forward model strategy.

Results: There were included 449 registries. One hundred an sisteen of them were SA. The fixed model showed a Chi-square=226.64 with 5 degrees of freedom and a $P<0.0001$. Explanatory variable gender was excluded from the forward model strategy due to its lack of impact on the binary response variable. The Odds Ratio for PCR, PCT $>1.3 \mathrm{ng} / \mathrm{dL}$, age, neutrofils recount and body temperature were $1.0175,8.1588,0.5135,1.0001$ and 3.4147 , respectively. The model showed a sensibility of $68.1 \%$, specificity of $94.8 \%$, PPV of $82.2 \%$ and NPV of $89.5 \%$. The following table shows the full results of the logistic regression analysis: Standard error, Wald index, $\mathrm{p}$ value and $\mathrm{R}$ coefficients.

\begin{tabular}{|llllll|}
\hline Variable & Coefficient & E. E. & $\begin{array}{l}\text { Chi2- } \\
\text { Wald }\end{array}$ & G.F. & p-value \\
\hline PCR & 0.0174 & 0.0024 & 52.0092 & 1 & $<0.0001$ \\
\hline PCT & 2.0991 & 0.3407 & 37.9640 & 1 & $<0.0001$ \\
\hline Age & -0.6665 & 0.1326 & 25.2763 & 1 & $<0.0001$ \\
\hline $\begin{array}{l}\text { Neutrophils } \\
\text { recount }\end{array}$ & 0.0001 & 0.0001 & 14.9630 & 1 & 0.0001 \\
\hline T>37.9 & 1.2281 & 0.3286 & 13.9674 & 1 & 0.0002 \\
\hline Constant & -3.7227 & - & - & - & N.A. \\
\hline
\end{tabular}

Conclusions: Previous studies have demonstrated the usefulness of PCT measure for the diagnosis of SA however all of them have been based on joints which synovial fluid is quite easy to obtain for further analysis. Present study allows laying the ground for creation of future diagnostic models based on five clinical variables that could be useful on scenarios where the assessment by joint puncture is not available.

Disclosure of Interest: None declared

DOI: 10.1136/annrheumdis-2017-eular.4240

\section{SAT0572 LEVELS OF SERUM PROCALCITONINE AS DIAGNOSIS DISCERNING TOOL BETWEEN GOUT AND SEPTIC ARTHRITIS}

C.A. Guillen-Astete, M. Terán-Tinedo. Rheumatology Department, Ramon y Cajal University Hospital, Madrid, Spain

Background: In a previous study we demonstrated the potential usefulness of the procalcitonine serum measure in order to diagnose septic arthritis. This utility is quite important on the differential diagnosis of knee arthritis in patients with known gout or other inflammatory joint disease which can express as a monoarthritis.

Objectives: Due to that, this study aims to determine sensibility, specificity, positive predictor value and negative predictor value of procalcinonine sereum measure (PCT) in patients with knee arthritis for the diagnosis of septic arthritis.

Methods: We reviewed the registries of patients who consulted due to knee acute monoarthritis between 2013 and 2015 in which a PCT was obtained. Registries were grouped according to the final diagnosis (gouty arthritis only (group I) and septic arthritis with or without gout (group II). Based on a previous study, PCT value of $1.47 \mathrm{ng} / \mathrm{mL}$ was considered the cut point. Validation tests were applied fixed to demographic and clinical specific scenarios.

Results: Registries of 121 patients with gout and 47 patients with septic arthritis (SA) were included. From the 47 patients with SA, 9 were previously diagnosed by gout. All diagnosed were based on guidelines and clinical recomendations for SA and gout (Coakley et al. Rheumatology, 2006 and Zhang et al. Ann Rheum Dis, 2006).

Using the cut point of $1.47 \mathrm{ng} / \mathrm{mL}$, test validation results were as follows: sensibility of $87.2 \%$, specificity of $92.5 \%$, PPV of $82.0 \%$ and NPV of $94.9 \%$. Excluding patients with less than 48 hours of onset, there were 44 registries of patients with SA and 83 with gout. In this scenario the results of the validation tests were as follows: Sensibility of $93.1 \%$, specificity of $96.3 \%$, PPV of $93.1 \%$ and NPV of $96.38 \%$. Excluding all patients with body temperature above $37.5^{\circ} \mathrm{C}$ there remained 11 with SA and 81 with gout. In this scenario the results of the validation tests were as follows: Sensibility of $72.7 \%$, specificity of $95.1 \%$, PPV of $66.6 \%$ and NPV of $96.2 \%$.

Conclusions: This is the first study aimed to validate previous observations about the usefulness of PCT determination in patients with acute knee monoarthritis. Altough the global results in the validation test are a bit inferior that our original observations in a shorter pilot study in terms of sensibility, the NPV remains over $95 \%$ in scenarios where the differential diagnosis could be harder to establish such as recent onset flares or cases without an associated febrile syndrome.

Disclosure of Interest: None declared

DOI: 10.1136/annrheumdis-2017-eular.4289 